\title{
ATM Transaction Status Feature Analysis and Anomaly Detection
}

\author{
Yunhui Zeng ${ }^{1}$, Ziyi Zhang ${ }^{1}$, Hongfei Guo ${ }^{2}$, Yilin $\mathrm{Chen}^{1}$, Shiyue Shen ${ }^{3}$, Longfei Zhu ${ }^{1}$, Bingjie $\mathrm{Yu}^{1}$ \\ ${ }^{1}$ College of Intelligent Science and Engineering, Jinan University, No. 206, Qianshan Road, Xiangzhou District, Zhuhai \\ City, Guangdong Province, China \\ ${ }^{2}$ College of Internet of Things and Logistics Engineering, Jinan University, No. 206, Qianshan Road, Xiangzhou \\ District, Zhuhai City, Guangdong Province, China \\ ${ }^{3}$ Institute for Economic and Social Research, Jinan University, No. 601, West Huangpu Avenue, Tianhe District, \\ Guangzhou City, Guangdong Province, China \\ Correspondence: Hongfei Guo, College of Internet of Things and Logistics Engineering, Jinan University, China.
}

Received: December 4, 2018

doi:10.11114/set.v6i1.3829
Accepted: January 27, 2019 Online Published: January 31, 2019

URL: https://doi.org/10.11114/set.v6i1.3829

\begin{abstract}
In this paper, based on ATM transaction status analysis and anomaly detection problem, by analyzing the transaction statistics of a bank ATM application system, the characteristic parameters of ATM transaction status are extracted and analyzed, then a set of targets are designed. An abnormal monitoring scheme that can promptly and accurately alarm the four abnormal situations in which the trading volume is steep, the transaction failure rate is increased, the transaction processing is slow, and the transaction response time is too long. Firstly, the transaction data is divided to distinguish between working days, non-working days, trading volume troughs, and normal trading periods, to avoid data interference between different time periods and to take into account the data discontinuity. The characteristics of the anomaly data were identified by K-Means Clustering Analysis. Then the data is analyzed by B-P Neural Network method, the change rule of ATM transaction status with time is obtained. According to this rule, the ATM transaction status is judged, and the abnormal situation is alarmed in time. Finally, this paper increases the amount and type of data collected, then increases the influencing factors such as ATM popularity, holidays, transaction types into the model, uses the existing transaction data before and after the Spring Festival to verify, in order to obtain a more realistic monitoring and early warning program. The transaction status anomaly monitoring scheme designed in this paper not only can correctly judge but also timely alarm the financial aid equipment failure scenarios, so that the security of the financial self-service equipment trading system is guaranteed.
\end{abstract}

Keywords: ATM Transaction Status, Abnormal Detection, Characteristic Parameters, K-Means Clustering Analysis, B-P Neural Network

\section{Introduction}

As the banking industry has increased the deployment of ATM equipment, the use rate of financial self-service devices such as ATM is getting higher and higher. Once the state of ATM is abnormal, it will not only affect people's normal production in life, but also initiating transaction security issues that cannot be ignored. Therefore, real-time monitoring of the ATM transaction status and timely detection of the abnormal state of the ATM trading system has become a common concern of the whole society. At present, there is not much research on the status of ATM transactions in China. From the overall perspective, the academic community mainly conducts information aggregation and data analysis on the three indicators of transaction volume, transaction success rate and response time, capturing the entire front-end and back-end overall. The operating conditions of the application system have obtained the trading characteristics of different time, it is better to predict the abnormal operation and failure of the ATM trading system, but it has not designed a complete anomaly detection system. For example, Chang Huihua and others in the study of the transaction data generated by the ATM trading system, the transaction volume, transaction success rate and response time characteristics analysis, using the variance analysis method and $3 \sigma$ thought to mark the abnormal point, to achieve the failure correct judgment of the scene, but the alarms are not analyzed in combination with the factors affecting life, such as holidays and transaction types (Chang Huihua,2018). Fei Jizhong summarized the faults of 9 major categories and 40 ATM systems according to the ATM usage of ICBC Branch of Shandong Province Weihai Branch (Fei Jizhong\&Wang jian,1999). The faults were classified into 5 categories, but did not design a comprehensive monitoring model to 
monitor faults. Hu Dingxian and others directly use the ATM network tester to access the measured end, run the traffic test function, automatically locate all transaction activities, identify relevant virtual channel identifiers and virtual path identifiers, monitor the error cells and traffic congestion (Hu Dingxian\&Xiong Wei,2002). evaluate the health of the ATM network. However, the tester must adapt to the different interface types selected and must meet the test requirements of the performance specifications of the ATM network under test. If the interface type cannot be adapted, the solution will not be implemented. Wang Chengliang adopts the product data management combined with the failure mode and the impact analysis method to analyze the financial product composition information, product function information and maintenance information, integrates the diagnostic knowledge acquired during the product design phase to diagnose, so that the product design, diagnosis and maintenance form an organic whole improves the diagnostic efficiency and accuracy (Wang Chengliang\&Gu Minjie,2011). However, this method cannot manage massive amounts of information, cannot update information in real time and deduct.

In this paper, the abnormal situation of the transaction is precisely defined, and the clustering analysis method is used to identify the abnormal data. Then, according to the location of the data, the emergency level of the abnormal situation is divided, the stratification is alarmed to obtain a more accurate early warning model. Finally, this paper increases the amount of data collected and adds factors such as ATM popularity, holidays, and transaction types to the model, verifies the existing data before and after the Spring Festival, which is more in line with the actual production and life needs.

\section{Method}

\subsection{Data Acquisition and Assumptions}

The original data of this paper is derived from "Shenzhen Cup" Mathematical Modeling Challenge, 2017 (Gao Zexu,2014). After consulting the Guangdong Organizing Committee to learn that the data derived is from 2017, this paper distinguishes between working days and non-working days and processes the data during the Spring Festival separately. For ease of analysis and understanding, this paper makes the following assumptions about the model:

(1) The data given in the competition is true and authentic;

(2) Assume that there is no significant difference in trading conditions between the corresponding working days and non-working days in each month in the short term;

(3) Assume that there is no significant difference in trading conditions between other holidays except the Spring Festival and the normal non-working days;

(4) Assume that abnormal data is only accounted for a little of the total sample.

\subsection{Model Establishment and Solution}

\subsubsection{Judgment of Working Days and Non-Working Days}

According to the notice of "Regulations on Some Holidays in 2017" issued by the General Office of the State Council of China(http://www.chinanews.com/gn/2016/12-01/8081085.shtml), the working days and non-working days can be distinguished. Moreover, the trading conditions during the Spring Festival is quite different from those in the normal working days and non-working days. Therefore, this paper does not attribute it to workdays or non-working days. The specific working days and non-working days are shown in Table 1.

Table 1. List of working days and non-working days

\begin{tabular}{|c|c|c|c|c|c|c|}
\hline \multicolumn{5}{|c|}{ Working day } & \multicolumn{2}{|c|}{ Non-working day } \\
\hline February $3^{\text {th }}$ & February $20^{\text {th }}$ & March $8^{\text {th }}$ & March $24^{\text {th }}$ & April $12^{\text {th }}$ & February $5^{\text {th }}$ & March $19^{\text {th }}$ \\
\hline February $4^{\text {th }}$ & February $21^{\text {th }}$ & March $9^{\text {th }}$ & March $27^{\text {th }}$ & April $13^{\text {th }}$ & February $11^{\text {th }}$ & March $25^{\text {th }}$ \\
\hline February $6^{\text {th }}$ & February $22^{\text {th }}$ & March $10^{\text {th }}$ & March $28^{\text {th }}$ & April $14^{\text {th }}$ & February $12^{\text {th }}$ & March $26^{\text {th }}$ \\
\hline February $7^{\text {th }}$ & February $23^{\text {th }}$ & March $13^{\text {th }}$ & March $29^{\text {th }}$ & April $17^{\text {th }}$ & February $18^{\text {th }}$ & April $2^{\text {th }}$ \\
\hline February $8^{\text {th }}$ & February $24^{\text {th }}$ & March $14^{\text {th }}$ & March $30^{\text {th }}$ & April $18^{\text {th }}$ & February $19^{\text {th }}$ & April $3^{\text {th }}$ \\
\hline February $9^{\text {th }}$ & February $27^{\text {th }}$ & March $15^{\text {th }}$ & March $31^{\text {th }}$ & April $21^{\text {th }}$ & February $25^{\text {th }}$ & April $4^{\text {th }}$ \\
\hline
\end{tabular}

\subsubsection{Judgment of the Trading Volume Trough Period and the Trading Volume Normal Period}

Within one day, the trading volume also has a trough period and a normal period. In order to distinguish them, the trading volume trough period and the trading volume normal period are defined as follows: the time period corresponding to the trading volume of 0 to half of the peak value is the trading volume trough period, and the time period corresponding to half of the peak value of the trading volume to the peak value is the trading volume normal 
period. Then find the two critical values of each day, perform arithmetic averaging on the peak and trough values in all sample data, objectively obtain the peak and trough periods in one day. The specific formula is as follows:

$$
\begin{gathered}
\alpha=\frac{\sum_{i=0}^{i} \alpha_{i}}{i} \\
\beta=\frac{\sum_{i=0}^{i} \beta_{i}}{i}
\end{gathered}
$$

Among them, $\alpha_{i}$ indicates the time when the day $i$ is about to become the trading volume normal period, and $\beta_{i}$ indicates the time when the day $i$ is about to become the trading volume trough period. The trend of trading volume in one day is shown in Figure 1.

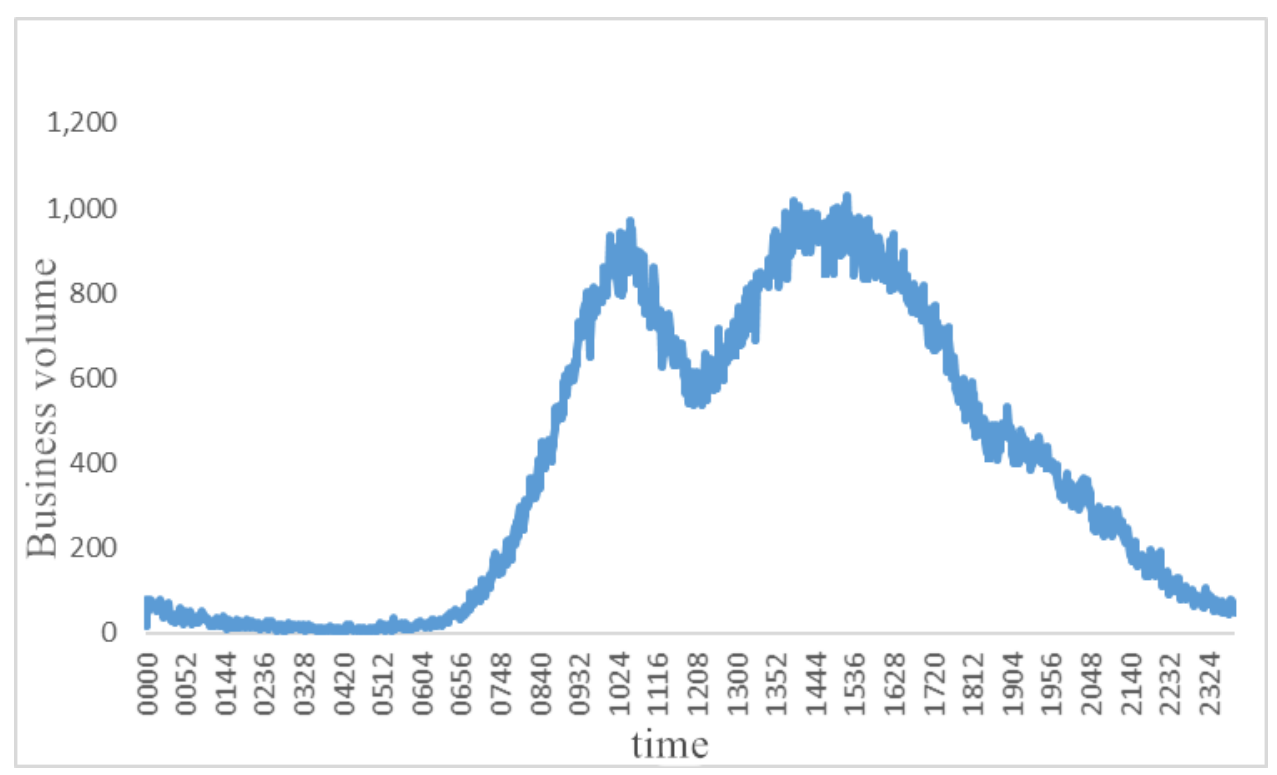

Figure 1. Trend of trading volume in one day

From the calculation of the average and Figure 1, within the allowable error range, the trading volume normal period of the working days is generally started at 8:04, and the trading volume trough period is started at about 20:07. Similarly, in non-working days, the trading volume normal period is generally started at $8: 12$, and the trading volume trough period is started at about 20:07.

\subsubsection{Using K-means Cluster to Eliminate Abnormal Data}

At present, the detection algorithms for abnormal data mainly include methods based on statistics, methods based on distance and methods based on cluster. A disadvantage of abnormal data detection method based on statistics is that it needs to know the data distribution or the approximate distribution of known data (Wu Jingfeng,2017). However, the distribution of real data is difficult to obtain. Therefore, when there is no specific test, the method cannot ensure that all abnormal data is found. Although the abnormal data detection method based on distance is widely used at present, it is very sensitive to the selection of parameters, which makes it highly unstable in the selection of abnormal data. The basic idea of cluster analysis is to classify the data according to the similarity degree of each pattern feature. Data with similar pattern features is classified into one class, and with non-similar pattern features is classified as another class. Combined with the characteristics of the discontinuity of the data used in this paper, after comparing the advantages and disadvantages of the three methods, this paper will identify the unusual data by the K-means cluster. Proceed as follows:

(1) Standardize data:

Since the data units are inconsistent, different data needs to be formatted to remove the influence of the data dimension within the specified range. In this paper, the z-score method is used to standardize the data according to the mean and standard deviation of the original data, so that the processed data conforms to the standard normal distribution. That is, the mean value is 0 , and the standard deviation is 1 . The specific operation is shown as formula (3). 


$$
\chi^{*=} \frac{\chi-\mu}{\partial}
$$

Among them, $x *$ is the new data obtained by standardization, ${ }^{\mu}$ is the mean of all sample data, $\partial$ is the standard deviation of all sample data, and $\chi$ is the original data that is not standardized. After that, the trading volume, success rate and response time are standardized to obtain three standardized variables: $\mathrm{Z}$ trading volume, $\mathrm{Z}$ success rate and $\mathrm{Z}$ response time.

(2) Cluster analysis of standardized variables

a. Initial classification

In this paper, $\mathrm{k}$ objects in the data space are selected as the initial cluster centers, and each object represents a class center. According to their Euclidean distance from these cluster centers and the nearest distance criterion, the sample data is assigned to the class represented the most similar cluster center. Among them, the Euclidean formula used to represent the similarity between different samples $x_{i}$ and $X_{j}$ is as formula (4):

$$
d\left(x_{i}, x_{j}\right)=\sqrt{\sum_{k=1}^{n}\left(x_{i k}-x_{j k}\right)^{2}}
$$

\section{b. Cluster analysis}

This paper uses K-means cluster to standardize scalar cluster. It is necessary to consider the effect on the authenticity of the results of the separation and the cluster of the three variables. According to the correlation analysis of the three variables, it is known that there is a negative correlation between the trading volume and the response time. If the three variables are analyzed one by one, the negative correlation will affect the result of cluster. Therefore, this paper deals with the three variables of trading volume, success rate and response time at the same time. Since the aforesaid assumption is that the abnormal data is only a small part, while selecting the number of clusters $K, K$ is gradually increased from 2 until the total number of small cluster sets obtained tends to be stable, and abnormal data is obtained according to the corresponding time points. The number of observations corresponding to each class of cluster in $\mathrm{K}$-means is shown in Table 2.

Table 2. The number of observations corresponding to each class of cluster in K-means

\begin{tabular}{l|ll}
\hline & 1 & 1.000 \\
Clustering & 2 & 4.000 \\
& 3 & 2818.000 \\
\hline Effective & 0 & 2823.000 \\
\hline Missing & 0 & .000 \\
\hline
\end{tabular}

c. Repeat iteration to determine the value of $\mathrm{k}$

Calculate the sum of the squares of the distances $J(C)$ from each point in the class to the cluster center $u_{i}$ and determine whether the value of the cluster center is changed according to the result. If it does not change, the cluster ends; otherwise, change the value of $K$ and re-cluster. The formula is as follows:

$$
J(C)=\sum_{k=1}^{K} \sum_{i=1}^{n} d_{k i}\left\|x_{i}-u_{i}\right\|^{2}
$$

d. Curve fitting to determine characteristic parameters

Curve fitting is a process method that using a continuous curve to describe or analogy the group function of discrete point on plane. Since the sample data is discrete and the number of the data is large, it is more suitable to analyze the characteristic parameters by curve fitting.

After eliminating the abnormal data according to the cluster method, the correct data in the sample is selected and is divided into two types: working days and non-working days. Then the curve is fitted, the result obtained is taken as the characteristic parameter of the trading state. For business volume:

In order to analyze the law of data variation more accurately and fit the data points with smoother curves, this paper uses the Smoothing Spline Interpolation Method to fit the business volume, that is, a third-order polynomial is used to 
model each segment between adjacent data points. While curve fitting, this paper uses MATLAB and try to call the following functions to fit the data:

$$
\begin{gathered}
\mathrm{p}=\operatorname{polyfit}(x, y, n) \\
{[p, S]=\operatorname{polyfit}(x, y, n)} \\
{[p, S, m u]=\operatorname{polyfit}(x, y, n)}
\end{gathered}
$$

Among them, formula (6) returns the coefficient of the polynomial $\mathrm{p}(\mathrm{x})$ of order $\mathrm{n}$, which is the best fit of the data in $\mathrm{y}$ (in the least squares formula). The coefficients in $\mathrm{p}$ are arranged in descending order, and the length of $\mathrm{p}$ is $\mathrm{n}+1$, as shown in formula (9):

$$
p(x)=p_{1} x^{n}+p_{2} x^{n-1}+\cdots+p_{n} x+p_{n+1}
$$

Formula (7) returns a structure $S$ that can be used as an input to polyval to obtain the error estimate the value. Formula (8) returns mu, which is a two-element vector containing the centered value and the scaled value. In the formula, mu (1) is mean $(\mathrm{x})$ and $\mathrm{mu}(2)$ is $\operatorname{std}(\mathrm{x})$. When using these values, polyfit places the center of $\mathrm{x}$ at zero and scales to have a unit standard deviation:

$$
\hat{x}=\frac{x-\bar{x}}{\sigma_{x}}
$$

This centralization and scaling transformation can simultaneously improve the numerical properties of the polynomial and fitting algorithms. In this way, it is found that the mean square deviation of the Smoothing Spline function is the smallest, that is, the smoothing average is the best. Therefore, the Smoothing Spline function is finally selected to smoothly fit the business. Figures 2 and Figure 3 are smooth curves of working days and non-working days respectively.

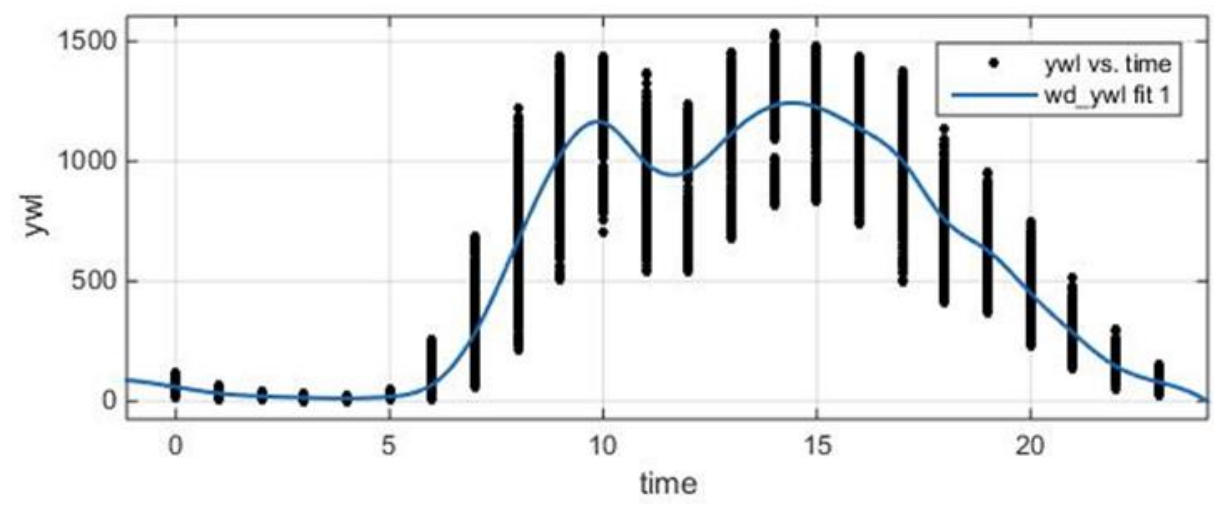

Figure 2. Smooth curve of trading volume of working days

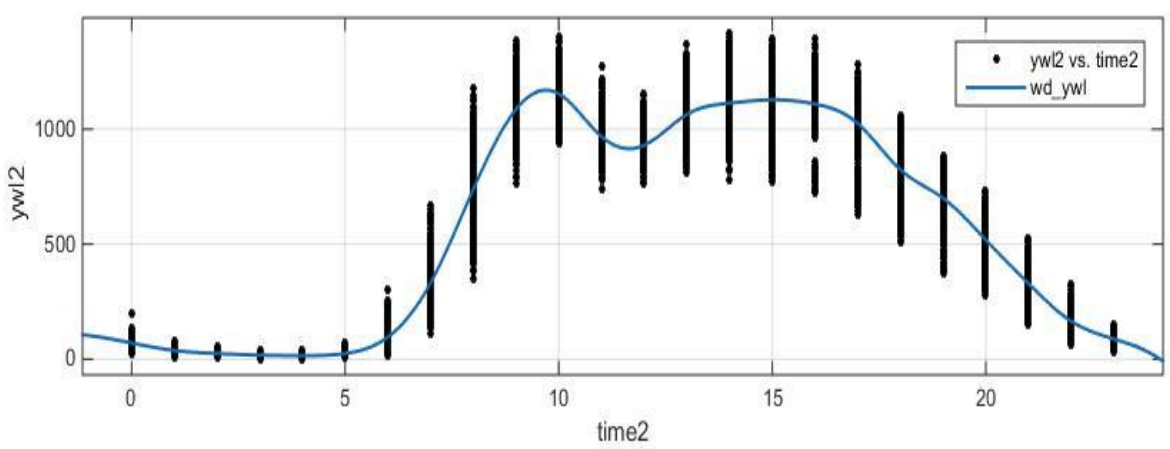

Figure 3. Smooth curve of trading volume of non-working days

Among them, the abscissa represents time (in hours) and the ordinate represents trading volume. If you want to get the trading volume at a certain moment, you can call the feval function in MATLAB to get the trading volume at the corresponding time.

For response time: 
After fitting the functions in the ATM toolbox, it is found that the RMSE (mean square deviation) of the Linear fitting function is the smallest, indicating that the fitting of the data is the best. Figure 4 and Figure 5 are the fitted curves about trading response times of the working days and non-working days respectively.

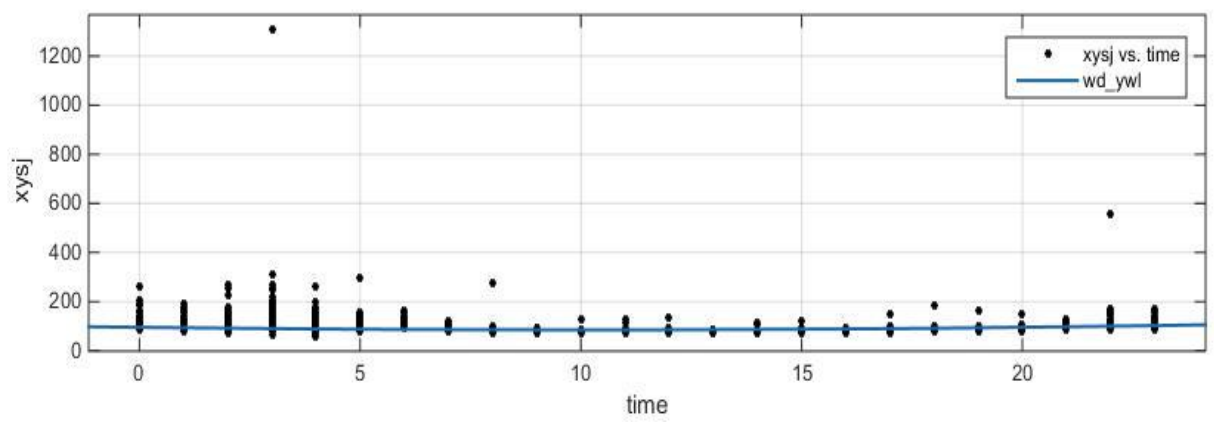

Figure 4. Fitted curve about response time of working days

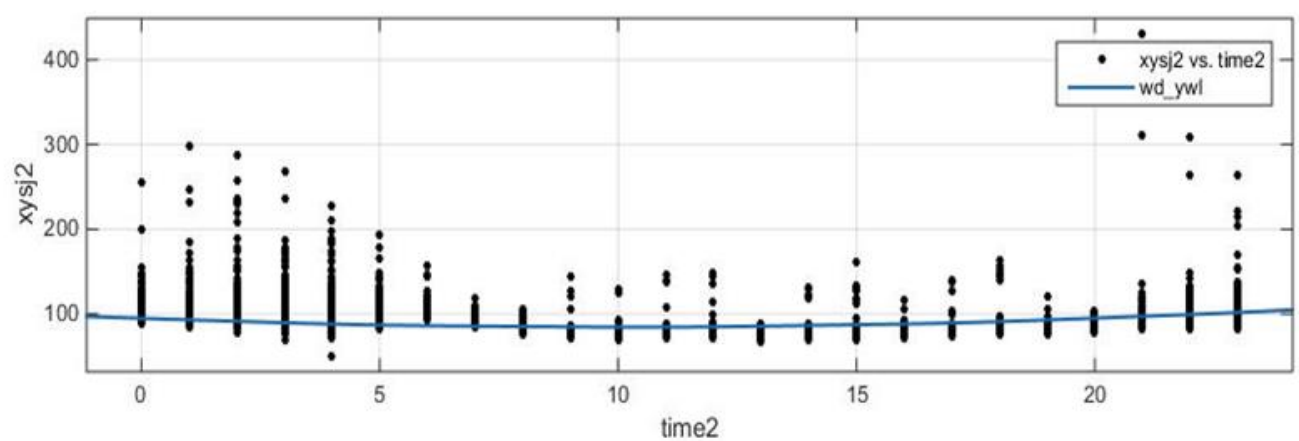

Figure 5. Fitted curve about response time of non-working days

From the figures, it can be seen that the linear relationship between trading response time and time is extremely insignificant and can be ignored. Therefore, this paper found that the normal trading response time in per hour is basically kept at $100 \mathrm{~ms}$.

For success rate:

Analogously to the above, it was found that the RMSE (root mean square deviation) was the smallest when the linear fitting function was selected, indicating that the data fits best at this time. Figure 6 and Figure 7 show the fitting curves about success rates of working days and non-working days respectively.

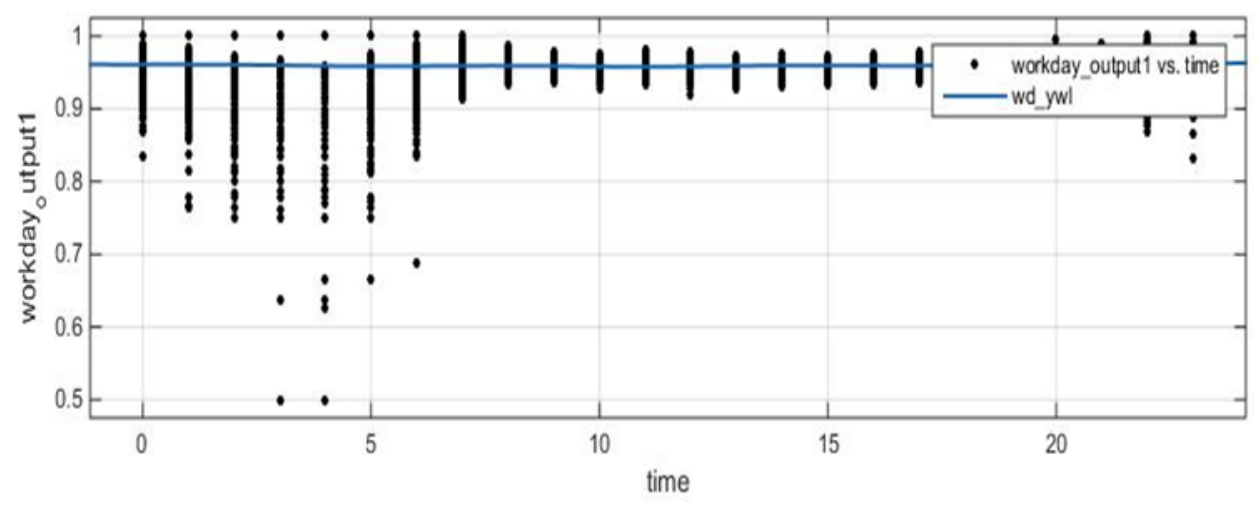

Figure 6. Fitted curves about success rate of working days 


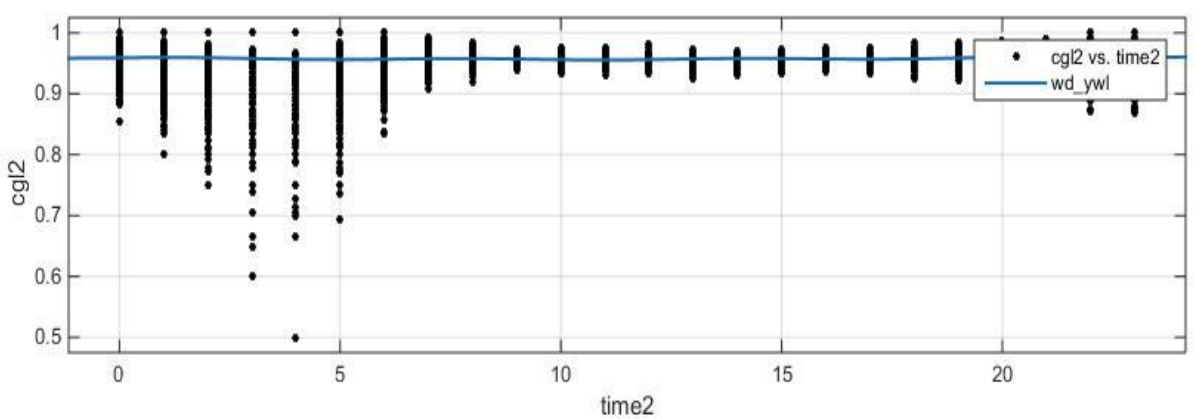

Figure 7. Fitted curves about success rate of non-working days

It can be seen from the figure that the linear relationship between success rate and time is not obvious and can be ignored. Therefore, it can be concluded that the success rate of normal data is basically maintained at $95 \%$.

\subsubsection{To Establish a Detection Scheme of the Exception of Transactions Status}

In this paper, B-P neural network method is used to establish the model, and to simulate and analyze the proposed ATM exceptions. The ATM neural network was constructed with the ATM business volume, the success rate and the data of response time of the branch in 4 months after the abnormal data were removed.

B-P neural network, that is, the learning process of error back-propagation error and back-propagation algorithm, is composed by the forward propagation of information and the back-propagation of error. By repeating the process of information forward propagation and error back propagation, the weights of each layer are constantly adjusted, that is, the process of neural network learning and training can be carried out until the error of network output is reduced to an acceptable level, or until the pre-set learning times. Due to the randomness and significance of abnormal values generated by faults, B-P neural network has the characteristics of self-learning, self-organization and self-adaptation, so this method is suitable for pattern recognition and classification. In this paper, due to the large number of samples, we can use the advantages and characteristics of B-P neural network to train the data and make real-time prediction of the future data distribution based on the trained data.

1) The Training of Neural Network

Firstly, to divide all the data, among them 80,000 data were used for the establishment and training of neural network and 40,000 data (including partial abnormal data) were used to verify the established neural network. The network will take two variables, "working day or not" and "time" as input, and the predicted business volume, the success rate and the response time as output. In addition, before the data analysis, since there is a large order of magnitude difference between response time and success rate, normalization is required. It not only can reduce the influence of different orders of magnitude between variables, to unify all variables in the interval $[-1,1]$, at the same time also can accelerate the training speed of neural network and the network convergence. The specific formula is as follows:

$$
y=\frac{\left(y_{\max }-y_{\min }\right) \times\left(t-t_{\min }\right)}{\left(t_{\max }-t_{\min }\right)}+y_{\text {min }}
$$

Among them, $y$ represents the value after normalization, $y_{\max }$ represents the maximum value after normalization, $\mathrm{y}_{\min }$ represents the minimum value after normalization, $t$ represents the input data, $t_{\max }$ represents the maximum value of input data, and $\mathrm{t}_{\min }$ represents the minimum value of input data.

Using the following network training parameters, B-P neural network can be trained, verified, simulated and predicted. The training parameters for ATM abnormal condition detection of B-P neural network are shown in the following table:

Table 3. Training parameters for abnormal condition detection of ATM in B-P neural network

\begin{tabular}{cccc}
\hline show & lr & Epochs & goal \\
\hline 52 & 0.01 & 200 & 0.001
\end{tabular}

Among them, show represents the number of steps displayed in the training process of neural network, that is, how many steps are taken at every interval to display the running results, lr represents the learning rate of neural network, epochs represent the maximum number of training steps required by the neural network, goal represents the target accuracy of the neural network. 
The mean square error (mse) of the simulation process was shown in the following figure, with the horizontal coordinate representing the network training step (epoch) and the vertical coordinate representing the network mse. The blue solid line represents the mse of network training, the red solid line is the mse of network prediction, and the intersection point of the two dotted lines is the best verification point of the neural network. Figure 8 is the schematic diagram of neural network training mse.

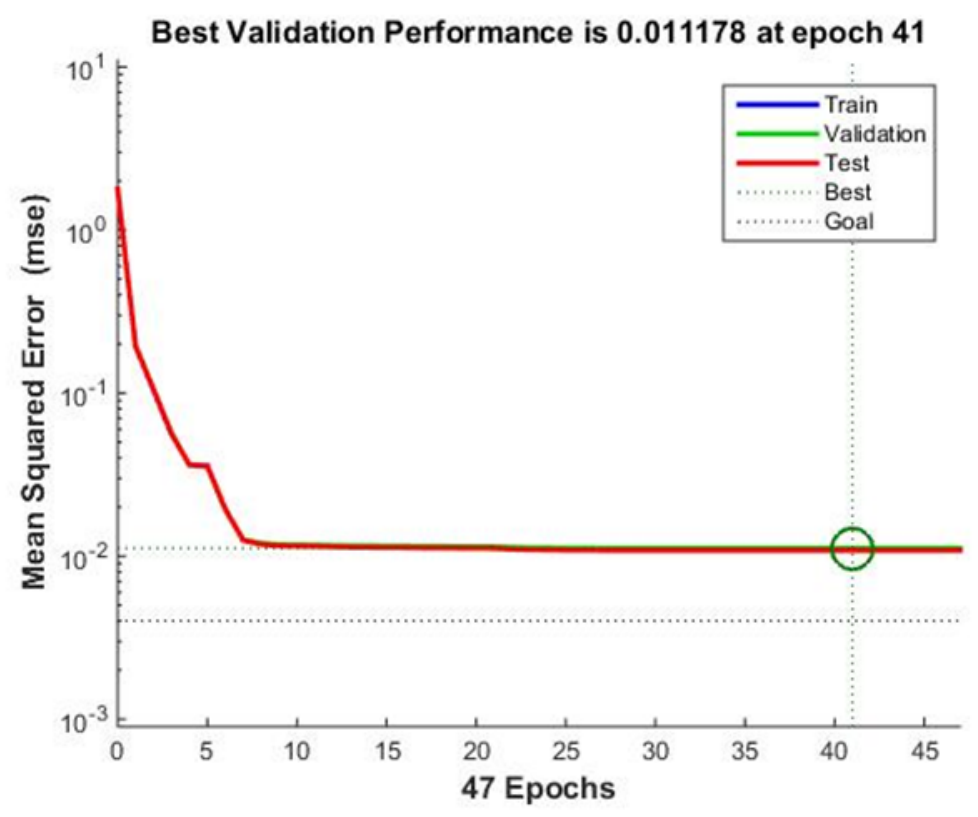

Figure 8. Schematic diagram of neural network training mse

The figure shows that, mse of the neural network is large at the initial stage. With the progress of neural network training, the neural network gradually converges and mse gradually decreases until it reaches a certain stable value. In the 47 steps neural network training process shown, when the number of network training steps reaches 52 , the mean square error has reached 0.001 and that is at the intersection of the two dotted lines. Therefore, this point is the best verification point of the neural network.

2) The Simulation of Neural Network

Secondly, through the simulation of neural network, the prediction of business volume, success rate and response time is drawn. The comparison between the prediction result and the actual result is shown as follows:

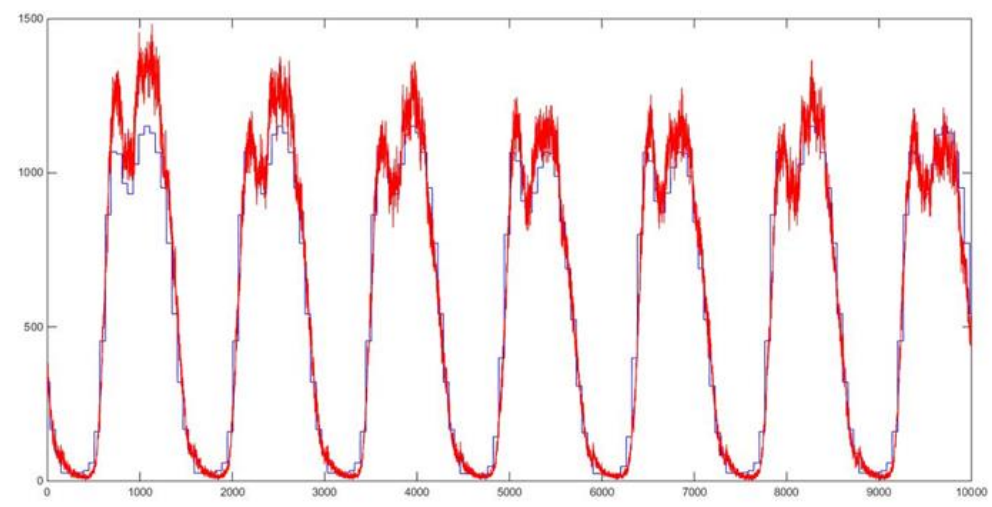

Figure 9. Comparison between the business volume forecast data of neural network and actual business volume 


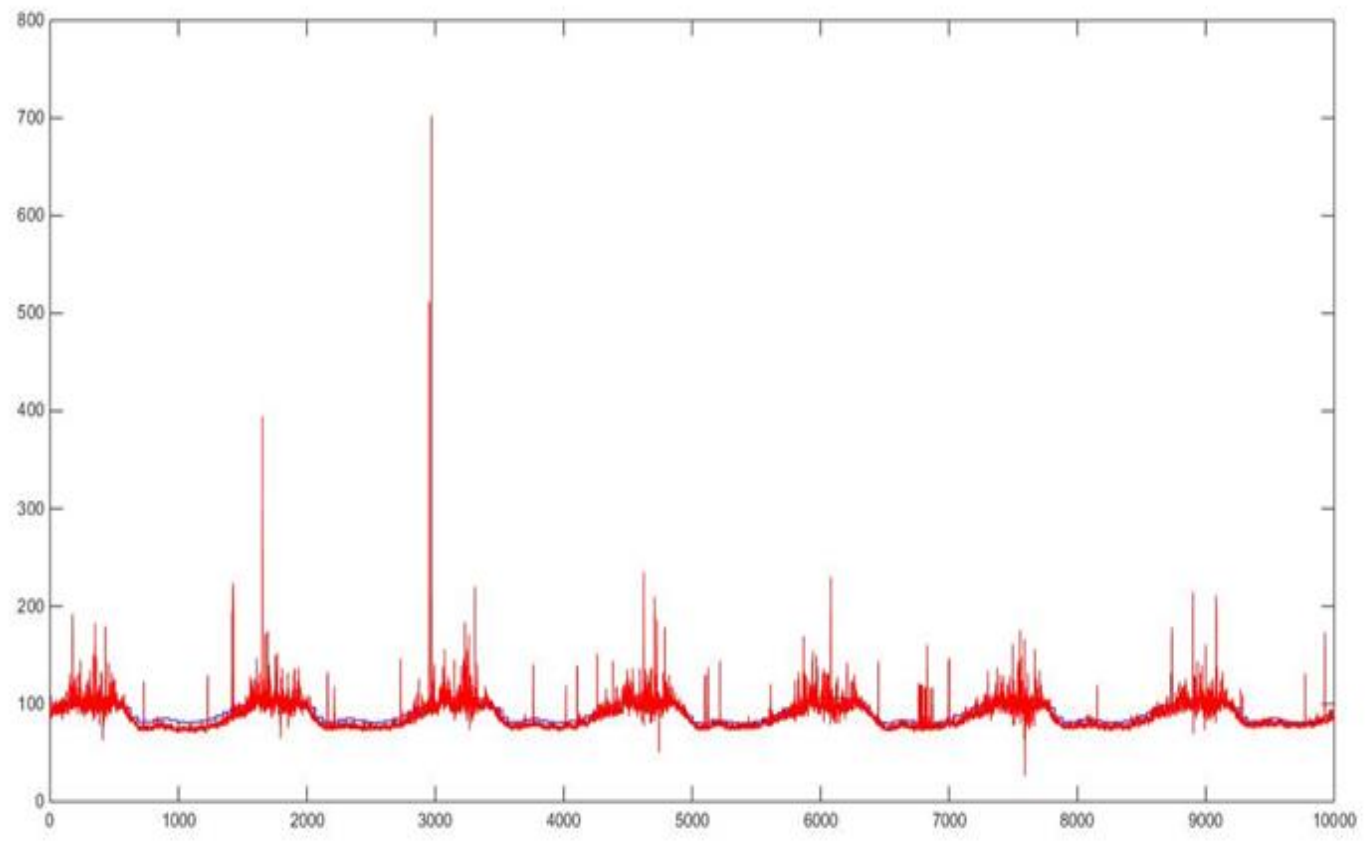

Figure 10. Comparison between the predicted response time of the neural network and the actual response time

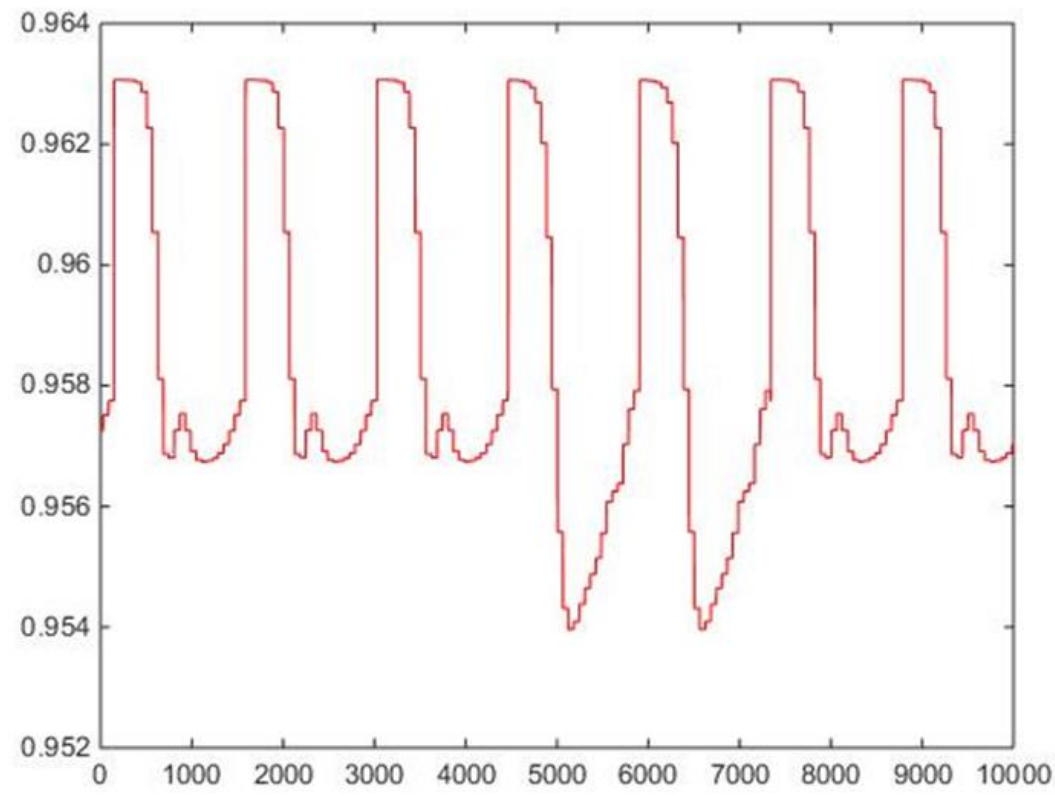

Figure 11. Success rate of neural network prediction

It is not difficult to see that the prediction result of the neural network is highly accurate and basically consistent with the actual data from the figure.

3) The Classification of Abnormal Condition

Using the confidence interval of business volume, success rate and transaction response time obtained in the following figure, the abnormal conditions are classified into columns, and different degrees of warning are given for different abnormal conditions.

Red alert: when the detected data deviates from the predicted data by more than $50 \%$, it indicates that the abnormal condition is particularly serious. 
Orange alert: when the deviation between the detected data and the predicted data is between $35 \%$ and $50 \%$, it indicates that the abnormal condition is serious.

Yellow alert: when the deviation between the detected data and the predicted data is between $20 \%$ and $35 \%$, it indicates that the abnormal condition is relatively serious.

Blue alert: when the deviation between the detected data and the predicted data is between $10 \%$ and $20 \%$, it indicates that the abnormal condition is not serious.

Green alert: when the deviation between the detected data and the predicted data is between 0 and 10 percent, it indicates that the trading status is roughly within the normal range.

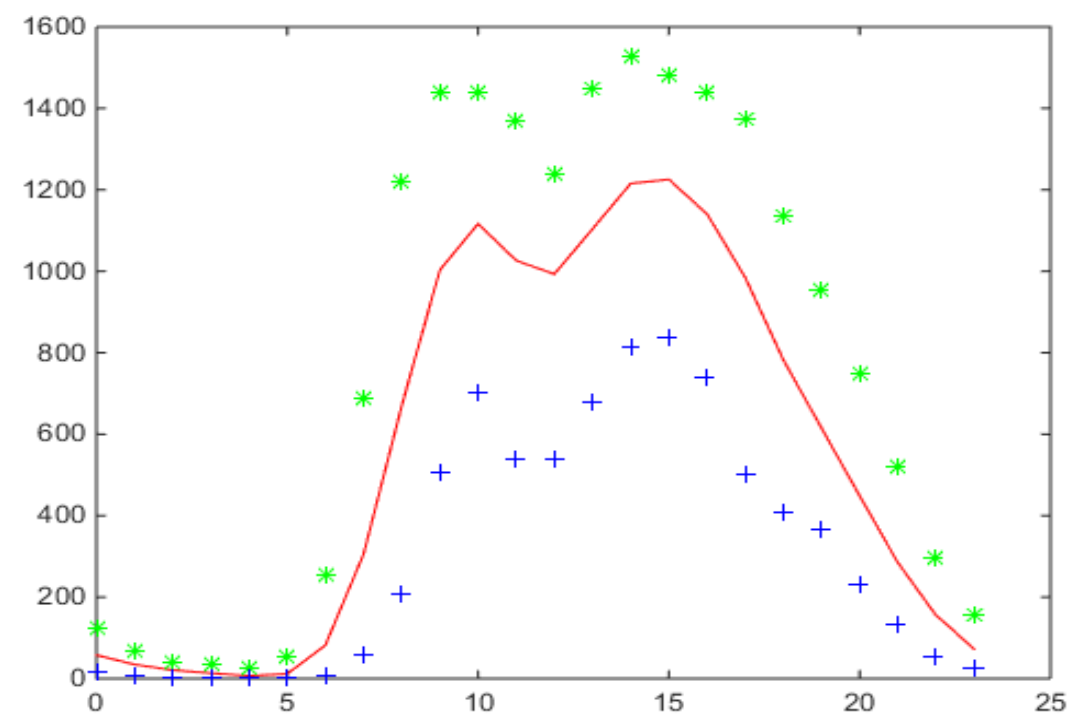

Figure 12. Confidence interval of business volume

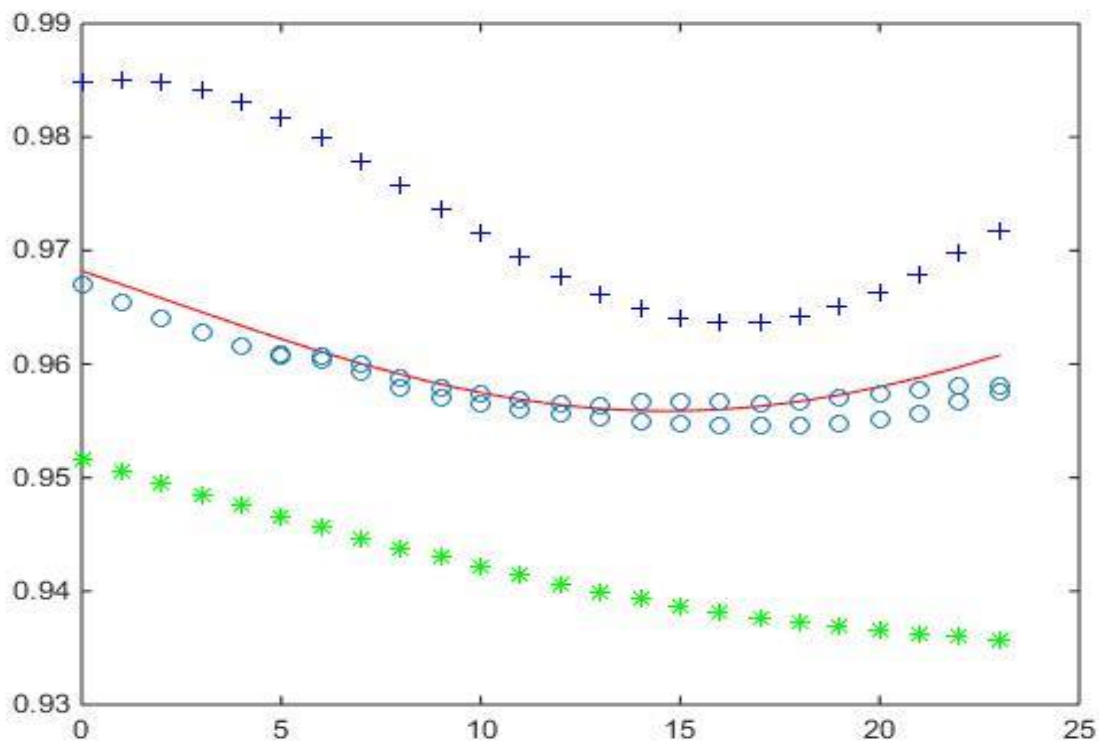

Figure 13. Confidence interval of success rate 


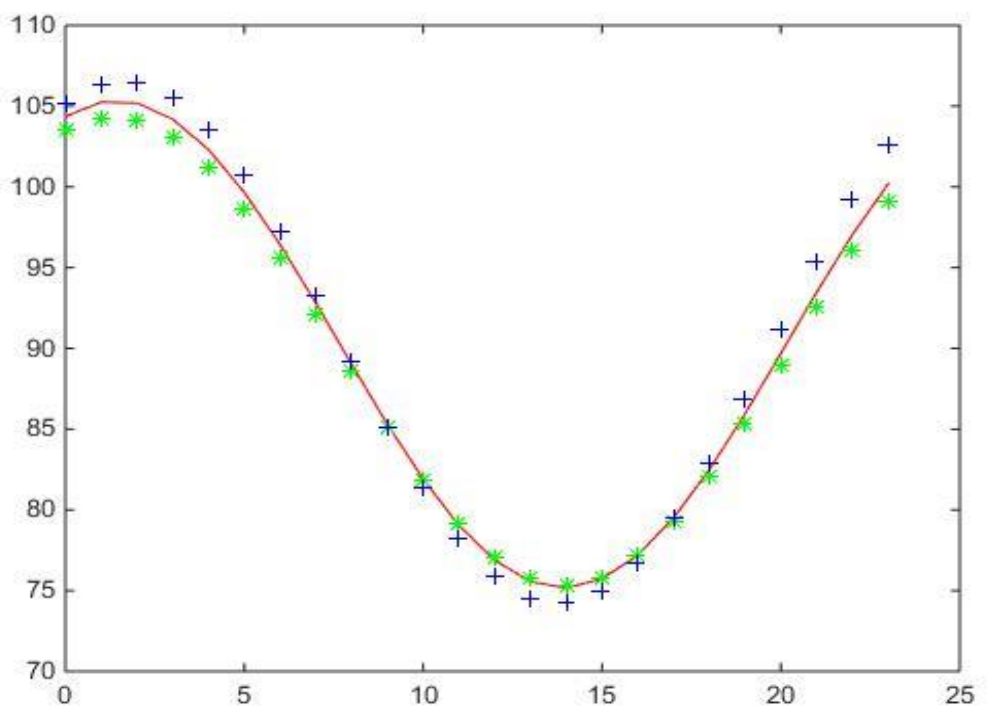

Figure 14. Confidence interval of response time

After obtaining the abnormal data, the fault category can be judged by the variable category where the exception occurred. In order to facilitate detection, binary indicators are defined for business volume, success rate and response time respectively. In case of failure, it will be 0 ; in case of normal, it will be 1 ; in case of all normal indicators, it will be output 111. If the branch side network transmission node fails or the front-end transaction fails to forward the request or the business volume drops sharply, the system will output the error code 011; If the branch side parameter data changes or is misconfigured or the failure rate of data center back-end processing increases or the transaction success rate is affected, the system will output error code 101. If the data center back-end processing system exception (such as the operating system CPU load is too large) causes slow transaction processing or affects the transaction response time, the system will output the error code 110.

\subsubsection{To Improve the Ability of Early Warning of Abnormal Values}

This paper proposes two ways to extend the data, and further optimization of the model.

1) Based on Extended Data Types

In daily life, ATM transaction response time is not only related to working days and non-working days, but also related to the following two factors:

One is the type of transaction. When ATM transaction types are different, the transaction response time will be greatly affected. For example, if the response time of deposit is assumed to be 5 seconds, the response time of query is 1 second, and the total transaction volume in the previous minute is 1000 , including 500 deposits and 500 queries. In the next minute, the transaction volume is still 1000, including 600 deposits and 400 queries. The two have the same transaction volume, but the transaction response time is different $(600 * 5+400 * 1-500 * 5-1 * 500) / 100=0.4$ seconds $=400$ milliseconds. At this time if we do not consider the transaction type, the data will be checked for exception by the model someday in the future, so this question will affect factors into consideration, this increase in the data collected by the data types of judgment, when to evaluate whether the abnormal data, consider due to trade the effects of different types, for the further optimization of the model.

Second is the transaction amount. Generally, when the transaction amount increases, the transaction response time will also increase under the same transaction volume. At this time, if the influence of transaction amount is not taken into account, a group of data will be detected to be abnormal under the two normal conditions of the same transaction volume and large difference in transaction amount, which greatly reduces the reliability of the model. Therefore, this paper takes this factor into account in the model, and further optimizes the model by extending the data to increase the collection of transaction amount data.

\section{2) Based on the Amount of Extended Data}

The data used in this paper are from January to April 2017. If the model is classified only according to working days and non-working days, it can neither reflect the special non-working days such as Spring Festival and National Day, nor show the time difference of system transaction status with the popularity of ATM. Therefore, based on the expansion of 
the amount of data collected, this paper increases the collection of data in other years to reflect the influence of special holidays and the popularity of ATM, and further optimization of the model.

Next, take the data around the Spring Festival as an example to illustrate that the increasing of data has a great impact on the model. In this paper, the B-P neural network method was used to divide the trading data around the Spring Festival into the periods of low trading volume and normal trading volume, and the results obtained during the Spring Festival were compared with those obtained in the non-working day model.

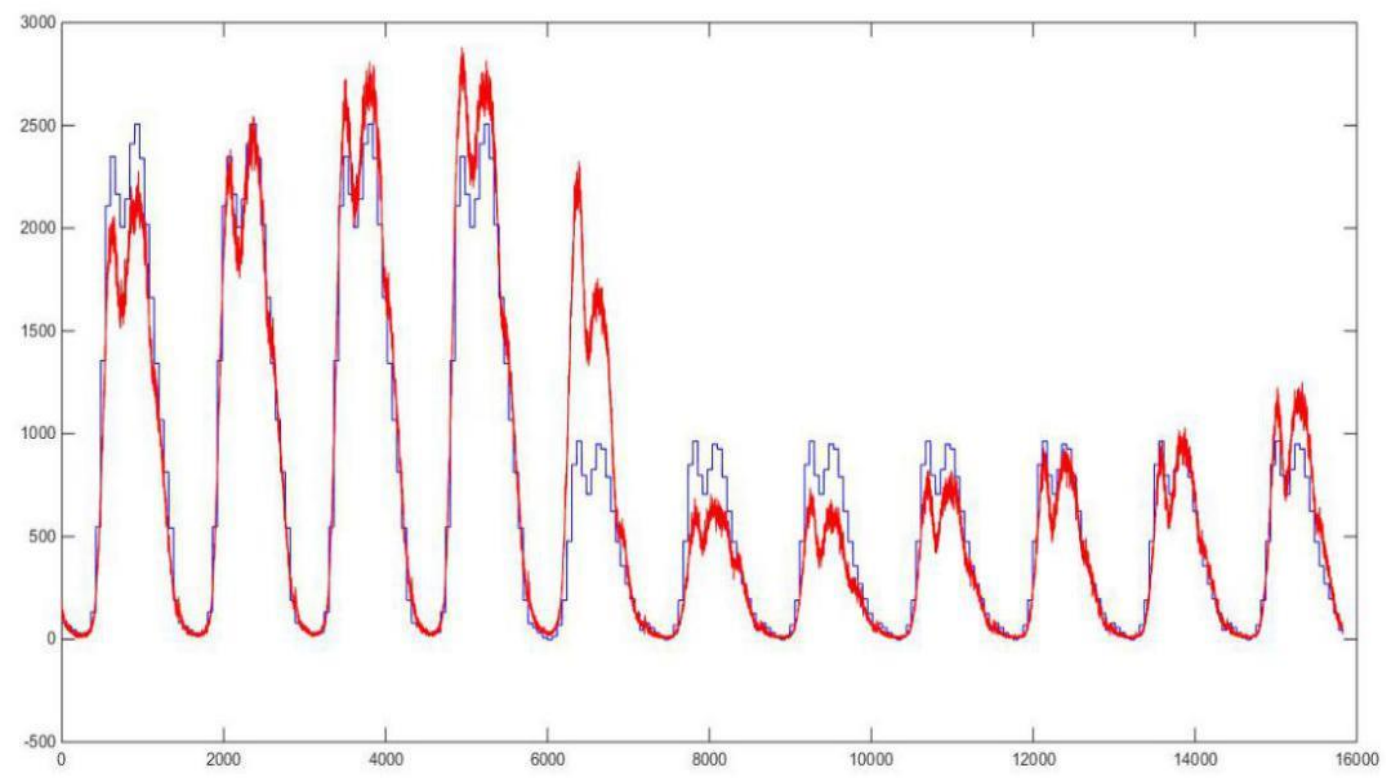

Figure 15. Separately considers the predicted value and the actual value of trading volume during the Spring Festival

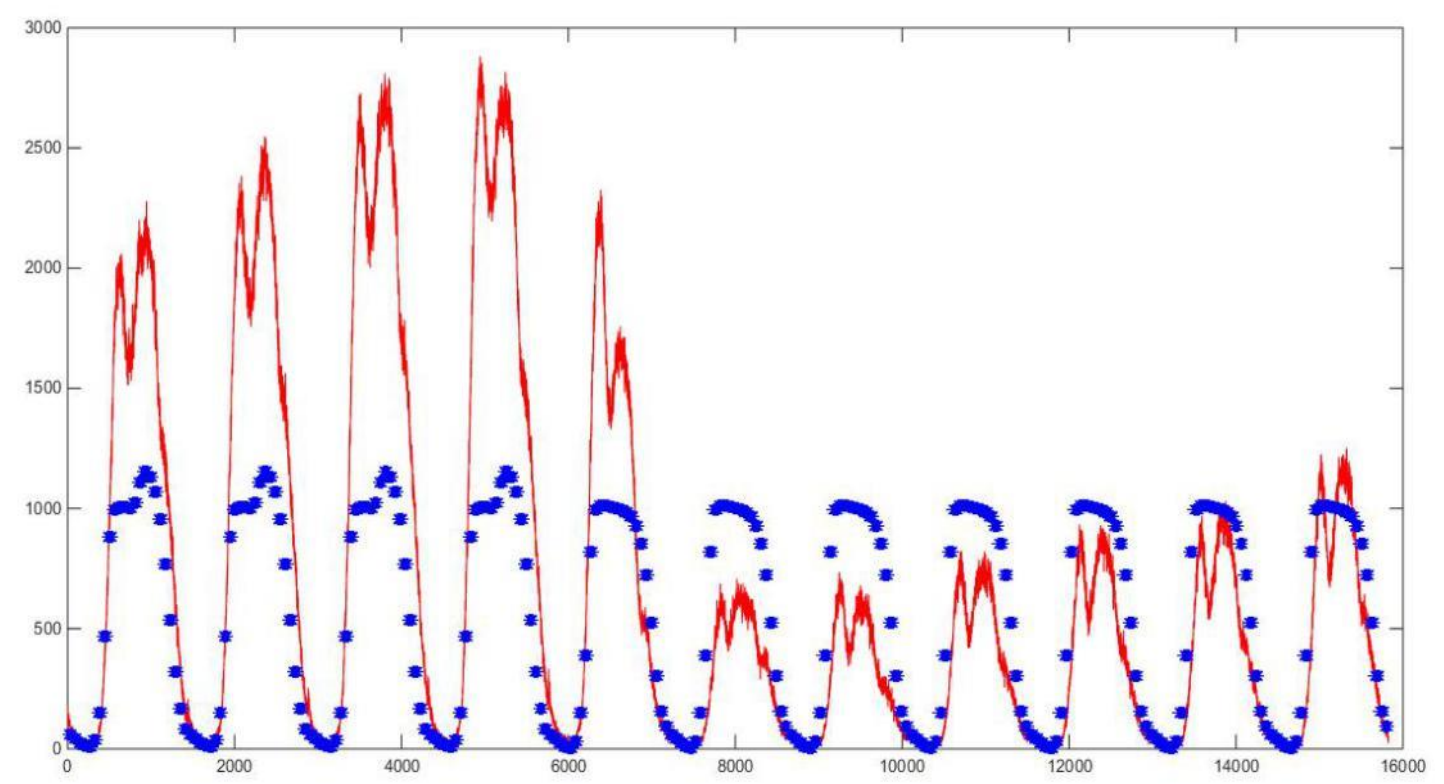

Figure 16. Considers the predicted value and the actual value of trading volume obtained during the Spring Festival as non-working days

As shown in figure 15, the blue line represents the predicted value of trading volume, and the red line represents the actual value of trading volume. According to the figure, when the Spring Festival period is treated as a special category 
separately, the predicted value of trading volume is highly consistent with the actual value. As shown in figure 16 , where the blue line represents the predicted value of trading volume and the red line represents the actual value of trading volume, it can be seen from the figure that when the Spring Festival is treated as a non-working day, there is a large deviation between the predicted value and the actual value.

Therefore, it can be seen that when the quantity of collected data is expanded and special holidays such as Spring Festival are considered separately in combination with daily life, the impact of time difference on the model can be weakened and the accuracy and application range of the model can be greatly improved.

\section{Results}

First of all, this article distinguishes between working days and non-working days according to the "Notice of the General Office of the State Council of China on Partial Holiday Arrangements for 2017", the arithmetic mean method is used to distinguish the business trough time period and the normal business time period, and the k-means clustering method is used for the data of each type of time period to distinguish the abnormal data. After that, the paper analyzes the curve of traffic volume with time by means of fitting. The transaction quantity, response time and success rate change time are used as the characteristic parameters of ATM transaction status, and B-P Neural Network method is used to analyze the data. Confidence interval for each feature parameter. Finally, according to the location of the data, the emergency level of the abnormal situation is divided, and the stratification is alarmed. In addition, the model obtained in this paper can be used in other systems including human-computer interaction and background processing, such as the campus internal campus card recharge system, self-service vending machine, automatic ticket vending machine, etc. The system ensures the security of people's transactions.

In terms of selecting research methods, this paper uses BP neural network to establish a model, which not only ensures that the model can be used for real-time online monitoring, but also the learning function will make the accuracy of the model increase gradually with time; K-means cluster analysis can be classified according to the similarity degree of each model to be classified. The similarity of pattern features is classified into one class, and the non-similarity is another class, which is consistent with the characteristics of data discontinuity in this paper.

However, when using the k-means clustering method, this paper re-classifies the calculated new hearts, and continues to calculate the center position of each type after classification, as a new focus, and repeats until the iteration termination condition is met. The operation is cumbersome. When the impact of the amount of data collected by the extension on the model is proposed, it cannot be verified by combining the model. In terms of the judgment of abnormal types, this paper only stays in the four kinds of abnormal categories, such as steep decline in transaction volume, increase in transaction failure rate, slow transaction processing, and long transaction response time. The classification of fault anomalies is not accurate enough, the range is too small.

\section{Acknowledgements}

This work was supported by the National Natural Science Foundation of China (51475095), the Fundamental Research Funds for the Central Universities (21618412), Key Project of Guangdong Natural Science Foundation (2016A030311041), 2015 Guangdong Special Support Scheme (2014TQ01X706), High-level Talent Scheme of Guangdong Education Department (2014-2016), the Guangdong Natural Science Foundation (2017A030313401).

\section{References}

Chang, H. H. (2018). ATM Trading State Feature Analysis and Anomaly Detection, [J]. Value Engineering.

Fei, J. Z., \& Wang, J. (1999). Experience in ATM Fault Diagnosis[J]. Computer and Credit Card, 1999(6), 32-35.

Gao, Z. X. (2014). ATM Fault Diagnosis Expert System Based on Neural Network[J]. China Financial Computer, 2014(10), 91.

Goldstein, M. (2012). Fast LOF: An expectation-maximization based local outlier detection algorithm[C]//nternational Conference on Pattern Recognition.IEEE,:2282-2285.

Hu, D. X., \& Xiong, W. (2002). Test and Fault Diagnosis of ATM Network[J]. Computer and Digital Engineering, 2002(4): 53-55.

Kruskal, W. H., \& Wallis, W. A. (1952). Errata: use of ranks in one - criterion variance analysis [J]. Journal of the American Statistical Association, 47(260), 583-621. https://doi.org/10.1080/01621459.1952.10483441

Li, E. Q. (2018). Construction method of fixed width confidence interval for zero-expansion Poisson distribution parameters [J]. Renmin University of China,

Lu, W. L. (2006). Research on real-time data prediction model of thermal power plant based on improved B-P neural network [J], Zhejiang University. 
Ma, G. J. (2017). Research on parallel coordinate visualization technology based on principal component analysis and k-means clustering $[\mathrm{J}]$, Xidian University,

Wang, C. L., Gu, M. J., \& Chen, J. J. (2011). PDM combined with FMEA for fault diagnosis of financial equipment [J]. Computer Systems Applications, 2011(2), 230-233.

Wang, Q, Wang, C., Feng, Z. Y., \& Ye, J. F. (2012). A Review of K-means Clustering Algorithms [J]. Xi'an Jiaotong University, 2012(07), 21-24.

Wu, J. F., Jin, Y. D., \& Tang, P. (2017). A Review of Monitoring Techniques for Data Anomalies[J]. Computer Science, 44(S2), 24-28.

Yang, W. (2002). Application of Artificial Neural Network Method in the Study of Moderate Scale of China's Foreign Debt [J], Tsinghua University

Ying, L. (2010). Abnormal data detection in agricultural search engines [D]. University of Science and Technology of China.

Yu, H. K., \& Lee, K. W. (2011). A study on the efficient operation of automated teller Machine (ATM) maintenance using simulation [C]// IEEE International Conference on Emergency Management Sciences, 520-524.

Yuwono, M., Moulton, B. D., Su, S. W., Celler, B. G., \& Nguyen, H. T. (2012). Unsupervised machine-learning method for improving the performance of ambulatory fall-detection systems[J].Biomedical Engineering Online, 11(1), 9. https://doi.org/10.1186/1475-925X-11-9

Zhang, C. (1998). Research on Intelligent Design of Mechanical System: Prediction of System Characteristics Using Neural Network [J], Shanghai Jiaotong University.

Zhang, C. Data stream clustering analysis and anomaly detection algorithm [D]. Fudan University.

Zhang, Y. L. (2002). The weighted method of the influence coefficient of neural network secondary learning and its example [J], Xi'an Jiaotong University.

Zimmermann, C. M., \& Bridger, R. S. (2000). Effects of dialogue design on automatic teller machine (ATM) usability:Transation times and card loss[J]. Bchaviour \& Information Technology, 19(6), 441-449. https://doi.org/10.1080/014492900750052697

\section{Copyrights}

Copyright for this article is retained by the author(s), with first publication rights granted to the journal.

This is an open-access article distributed under the terms and conditions of the Creative Commons Attribution license which permits unrestricted use, distribution, and reproduction in any medium, provided the original work is properly cited. 